\title{
RADIAL VELOCITIES FOR THREE DISTANT LMC GLOBULAR CLUSTERS
}

\author{
JESPER STORM ${ }^{1,2}$ and BRUCE W. CARNEY ${ }^{1}$ \\ ${ }^{1}$ Univ. of North Carolina at Chapel Hill \\ Dept. of Physics and Astronomy \\ Phillips Hall, $C B \# 3255$ \\ Chapel Hill, NC 27599, USA
}

\author{
${ }^{2}$ Copenhagen Univ. Observatory \\ Dster Voldgade 3 \\ $D K$-1350 Copenhagen $K$. \\ Denmark
}

ABSTRACT. In an attempt to investigate the large scale structure and dynamics of the old population of the LMC, we have obtained high precision radial velocities for three extremely outlying $\left(10^{\circ}-15^{\circ}\right.$ from the LMC center) globular clusters. Our results agree well with the results from Freeman et al. (1983) that the clusters move in a disk like system. Depending on the assumptions used we can put a lower limit to the LMC mass of about $0.5-2.5 \times 10^{10} M_{\odot}$

\section{Data and data reduction}

Radial velocities for two stars in cach of the clusters NGC1841, Reticulum and ESO121SC03 have been obtained using the $2.5 \mathrm{~m}$ DuPont telescope at Las Campanas Obscrvatory equipped with the echelle spectrograph and 2D-Frutti photon counting detector. The stars were all very faint with V magnitudes in the range $16^{m}-17^{m} .2$. Exposure times of the order 1 hour sufficed to give reliable velocitics.

The echelle data were reduced using IRAF to perform the basic preprocessing including the extraction and wavelength calibration of the individual orders. The cross-correlation was done using the Harvard XCOR package for IRAF. For each echelle spectrogram, 10 orders were extracted covering the wavelength region from $4750-5550 \AA$. The 10 resulting $1 \mathrm{D}$-spectra were then individually crosscorrclated with a sky template and a template from the star 47Tuc-C349 (a giant), each of which were observed each night. In several cases the sky template showed a bad corrclation with the object spectrum probably because of a bad match between the spectral type of the star and that of the Sun. These results were rejected.

The results are summarized in the table below. The position angles (PA) and the galactocentric velocitics $\left(V_{c l, c}\right)$ were detcrmined following Feitzinger and Weiss (1979) adopting their value for the effective solar apex and taking the LMC rotation center to be $(\alpha, \delta)=\left(5^{h} 21^{m} .1 ;-69^{\circ} 19^{\prime} .1 ; 1950.0\right)$ (Hartwick and Cowlcy (1988)).

\section{Discussion}

In fig. 1 and 2 these new points have been entered in the plots from Frecman et al. (1983) and it is evident that they agrec well with the previous data. The curve drawn in fig. 1 is solution 3 from that paper, based on the old cluster population (SWB classes V-VII, Scarle et al. (1980)). Looking only at the oldest clusters (SWB class VII) suggests that a slightly different solution is warranted for this population. Figure 2 shows that the assumption of a flat rotation curve is good even out to these large distances.

Since the ESO cluster is very close to the line of nodes and the clusters apparently follow the general rotation pattern of the LMC, this cluster can give important information about the mass of the LMC. Assuming $(m-M)_{L M C}=18^{m} .5$ gives a projected distance (which is close to the real distance because this cluster is almost on the line of nodes) from the LMC center of $8.5 \mathrm{kpc}$ for this cluster. Correcting the galactocentric velocity for a systemic velocity of the LMC of $26 \mathrm{~km} / \mathrm{s}$ (solution 3 ) and assuming a circu- 
lar orbit then gives a lower limit for the LMC mass of $5 \times 10^{9} M_{\odot}$. This number can be raised substantially if we believe that the clusters follow the general rotation of the LMC and that solution 3 from Freeman et al. (1983) is valid as indicated above. Correcting $V_{c l, c}$ for an inclination $i=27^{\circ}$ gives a circular velocity $V_{\text {circ }}$ of $110 \mathrm{~km} / \mathrm{s}$ leading to $M_{L M C, \min }=2.4 \times 10^{10} M_{\odot}$. Another approach which is based on all the available information, is to rely on the fitted model and assuming a flat rotation curve out to $15^{\circ}$ from the center, as indicated by these observations. The angular distance of $15^{\circ}$ converts to $15 \mathrm{kpc}$. Correcting $V_{\max }$ for solution $3(\approx 41 \mathrm{~km} / \mathrm{s})$ for an inclination of $i=27^{\circ}$ gives a circular velocity of $90 \mathrm{~km} / \mathrm{s}$ leading to a mass of $\approx 2.5 \times 10^{10} M_{\odot}$. The value of $i$ is not well determined though, and adopting a more conservative value of $i=45^{\circ}$ leads to mass estimates of $\approx 40 \%$ of the above or about $1 \times 10^{10} M_{\odot}$.

\begin{tabular}{|l|c|c|c|c|c|c|c|}
\hline Cluster & $\begin{array}{c}\text { RA } \\
(1950.0)\end{array}$ & $\begin{array}{c}\text { DEC } \\
(1950.0)\end{array}$ & $\begin{array}{c}\text { PA } \\
\text { degrees }\end{array}$ & $\begin{array}{c}\mathrm{d} \text { (center) } \\
\text { degrees }\end{array}$ & $\begin{array}{c}\text { Vrad } \\
\mathrm{km} / \mathrm{s}\end{array}$ & $\begin{array}{c}\text { Vcl,c } \\
\mathrm{km} / \mathrm{s}\end{array}$ & DELTA V \\
\hline Reticulum & $4: 35: 24$ & $-58: 56: 00$ & 329.2 & 11.52 & $235.0+-1.8$ & 28 & -9 \\
ESO121SC03 & $6: 01: 10$ & $-60: 31: 31$ & 30.4 & 9.74 & $310.8+-1.8$ & 76 & 10 \\
NGC1841 & $5: 11: 11$ & $-84: 04: 54$ & 181.0 & 14.77 & $204.3+-2.3$ & -1 & -3 \\
\hline
\end{tabular}

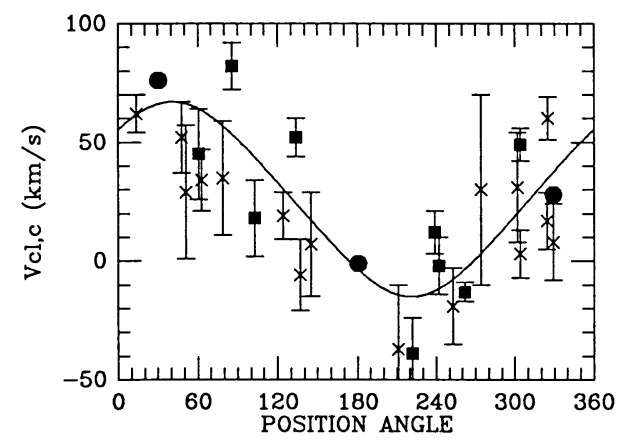

Fig.1 Galactocentric velocities $\left(V_{c l, c}\right)$ as a function of position angle. $\times=S W B$ class $V-V I$, $\mathbf{n}=$ SWB class $V I I, \bullet=$ new points

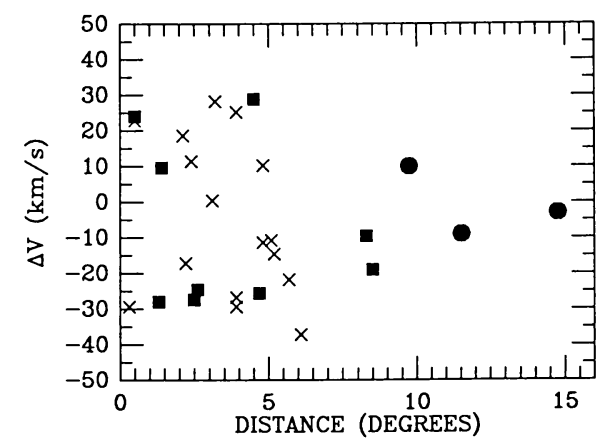

Fig.2 Velocity difference $(\Delta V)$ between observation and model versus $d$, the angular distance from the LMC rotation center. $\times=S W B$ class $V$ $V I, \mathbf{\square}=S W B$ class $V I I, \bullet=$ new points

\section{Conclusions}

These new measurements agree well with the picture put forward by Freeman et al. (1983) that the old globular clusters move in a disk-like system with a flat rotation curve. A more numerous pop-II tracer is clearly needed to assess the question of the existence of a dynamical LMC halo population.

From the ESO cluster alone we can infer a minimum mass of the LMC of $5 \times 10^{9} M_{0}$, and if we believe the above conclusion that the old population moves in a disk-like system with a flat rotation curve described by solution 3 , this estimate can reasonably be raised to $1 \times 10^{9} M_{\odot}$ if not more, depending on the value for the inclination of the disk.

\section{References}

Feitzinger, J.V. and Weiss, G. (1979), Astron. and Astrophys. Supp. 37, 575.

Freeman, K., Illingworth, G. and Oemler, A. (1983), Astrophys. Journ. 272, 488.

Hartwick, F.D.A. and Cowley, A.P. (1988), Astrophys. Journ. 334, 135.

Searle, L., Wilkinson, A. and Bagnuolo, W.G. (1980), Astrophys. Journ. 239, 803. 